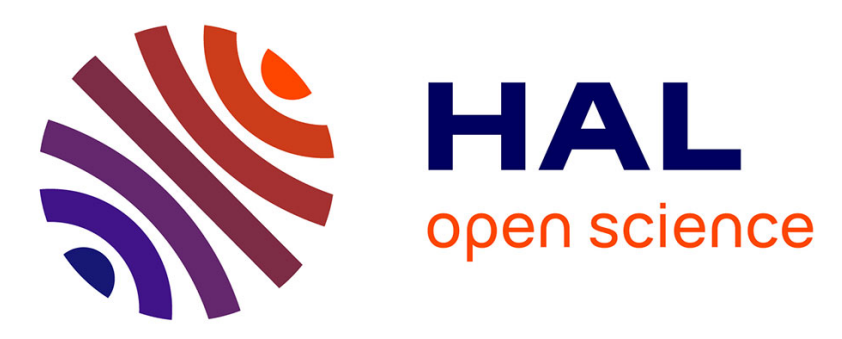

\title{
Massive MIMO Cooperative Communications for Wireless Sensor Networks: Throughput and Energy Efficiency Analysis
}

Nadjib Achir, Mérouane Debbah, Paul Mühlethaler

\section{- To cite this version:}

Nadjib Achir, Mérouane Debbah, Paul Mühlethaler. Massive MIMO Cooperative Communications for Wireless Sensor Networks: Throughput and Energy Efficiency Analysis. PIMRC 2014, Sep 2014, Washington DC, United States. hal-01092541

\section{HAL Id: hal-01092541 \\ https://inria.hal.science/hal-01092541}

Submitted on 8 Dec 2014

HAL is a multi-disciplinary open access archive for the deposit and dissemination of scientific research documents, whether they are published or not. The documents may come from teaching and research institutions in France or abroad, or from public or private research centers.
L'archive ouverte pluridisciplinaire HAL, est destinée au dépôt et à la diffusion de documents scientifiques de niveau recherche, publiés ou non, émanant des établissements d'enseignement et de recherche français ou étrangers, des laboratoires publics ou privés. 


\section{Massive MIMO Cooperative Communications for Wireless Sensor Networks: Throughput and Energy Efficiency Analysis}

\author{
Nadjib Achir \\ Universite Paris 13, Sorbonne Paris Cite, \\ L2TI (EA 3043) \\ F-93430, Villetaneuse, France \\ Email: nadjib.achir@univ-paris13.fr
}

\author{
Mérouane Debbah \\ SUPELEC \\ Alcatel-Lucent Chair on Flexible Radio \\ 3 rue Joliot-Curie \\ 91192 Gif Sur Yvette Cedex, France \\ Email: merouane.debbah@supelec.fr
}

\author{
Paul Muhlethaler \\ INRIA HiPERCOM, Rocquencourt \\ B.P. 105, 78153 Le Chesnay, France \\ Email: paul.muhlethaler@inria.fr
}

\begin{abstract}
The objective of this study is to analyze a new disruptive deployment of wireless sensors in order to cope with the explosive demand for bandwidth while taking into account energy consumption considerations. The work is grounded on the idea of massive network densification by drastically increasing the number of sensors in a given area in a Time Division Duplex (TDD) mode. Using ideas from the recent Massive MIMO technology (more than $\mathbf{4 0 0}$ antennas, without any modification of the network infrastructure), we transpose the idea to a massive deployment of sensors and show the benefits of such an infrastructure. This research is expected to provide the optimal deployment of massive wireless sensor networks in terms of cost/performance/complexity/energy efficiency trade-off and define the next generation wireless Machine-to-Machine (M2M) communication.
\end{abstract}

\section{INTRODUCTION}

In our modern world, more than half of the world's population lives in urban or sub-urban areas. Last year, the world's population reached 7 billion and current projections predict that it will exceed 9 billion by 2050, $70 \%$ of which will live in cities. This continuous expansion of urban populations, increases the need to efficiently design and control complex and interdependent urban infrastructures related to urban living, such as public safety, public transportation, traffic management, resource/energy utilization, interpersonal communications, social activities, and entertainment, etc. Providing such an efficient control of urban infrastructures greatly depends on the availability of and access to large amounts of information about urban environments and infrastructures. Data gathering is the key issue in order to efficiently monitor existing infrastructures or provide new urban services. Basically, we need to acquire data from a number of sources, process this huge amount of data, and then push or make these data available to city infrastructure managers and citizens in a user-friendly manner.

Currently, among all the available technologies, we believe that Wireless Sensors Networks (WSNs) are undoubtedly best suited to efficiently acquire and disseminate data on a large scale. Recent advances in Micro-Electro-Mechanical Systems (MEMS) technology, wireless communications, and digital electronics have enabled the development of low-cost, low-power nodes (also called sensor nodes) that are able to communicate over short distances. These sensor nodes consist of sensing, data processing units, and communication components. Consequently, a large number of companies are proposing new small devices which can monitor different phenomena such as temperature, humidity, vibration, pressure, and several other factors. These sensors can be deployed to build a Wireless Sensor Network (WSN). Thus, WSNs could be used to monitor the urban environment in real time, to facilitate automated control and to collect information for decision making. Nowadays, sensors are incorporated in most of our modern facilities, such as mobile phones, vehicles, buses, bus stops, bikes, etc. For example, mobile phones, with their increasing capabilities are used as voice communication devices but also as a sensing device able to collect and transfer data such as images, audio, GPS position, speed, etc. All these sensors could play an important role in providing a vast amount of dynamic information about their environment. Therefore wireless sensor networks could be a valid solution to urban monitoring problems by bringing new services for the city and for the citizens.

Over the last decade, WSNs have generated considerable enthusiasm within the networking research community. Many studies have been conducted in order to apply wireless sensor networks to a wide range of applications. The majority have focused on several networking issues, such as routing, MAC, data gathering and dissemination mechanisms. Unfortunately, few studies have been conducted on large-scale WSNs, where the number of nodes can reach thousands or even more, which is the case if we consider the context of smart cities. In fact, the few studies such as [1], [2], [3], [4], [5] that explicitly considered large-scale wireless sensor network are often optimized to meet the specific needs of the application and do not fully leverage the general network behaviors. Moreover, due to the intrinsic sensors characteristics including limited energy, limited communication range and a relatively large area of interest (urban areas), full connectivity could not be achieved.

To the best of our knowledge, the mainstream methods that could be used to solve the problem of information gathering in large-scale WSNs are the hierarchical routing protocols [6], [7], [8], [9], [10]. Indeed, in a hierarchical routing protocol, the 
sensor network is partitioned into several groups with different assignment levels. The nodes of the low levels are responsible for sensing and collecting the information from their environment, whereas the high level sensors are responsible for gathering the information from their low level sensors and then forwarding this information to the next high level. This hierarchical routing approach has proved to be more energy-efficient than a complete flat network. Unfortunately, this approach requires the deployment of higher energy nodes acting as cluster heads in order to gather, process and send the information from lower level nodes to higher level nodes. Since the cluster heads are acting as relays they will see their energy consumption fast increasing, unless they are given a high energy capability. In addition, the malfunctioning of the cluster head can lead to a strong system degradation in terms of performance. To overcome these limitations, we propose to use, in this paper, a distributed Massive MIMObased collaboration between the sensors [11], [12] in order to improve the performances of large-scale wireless sensor networks.

Conventional Multiple-Input Multiple-Output (MIMO) wireless communication, through the use of multiple antennas both at the transmitter and at the receiver sides, has the potential of multiplying the capacity of a single channel of bandwidth $W$ by the rank of the channel matrix. For sufficiently rich scattering, this rank is $r=\min (M, N)$, where $M$ and $N$ denote the number of transmitting and receiving antennas, respectively. MIMO has the capability of multiplexing $r$ independent channels, and therefore achieve an $r$-fold increase in system capacity. Recently cooperative MIMO has gained substantial research interest [13], [14] due to its capability to exploit conventional MIMO techniques for physically constrained mobile devices. In this case, the spectral efficiency for users is greatly improved when users share their antennas to jointly transmit and decode the data. In this paper, we focus on massive MIMO cooperative communications in the case of wireless sensor networks. The main idea is to characterize the performances of the system in terms of throughput/complexity/energy efficiency trade-off.

The reminder of this paper is organized as following: Section II describes the system model including the training and the transmission periods. At the end of this section we derive both the overall system throughput and energy consumption. Section III describes the simulation and results. Finally, section IV concludes the paper and discuss directions for our future works.

\section{SYSTEM MODEL}

Let $N$ be a set of sensors randomly distributed in a two dimensional field. We consider that the $N$ sensors are identical and equipped with one transmit antenna. In addition to the detection capability, each sensor is able to communicate in order to notify the sink of the event that has been detected. Basically, when an event is detected, each sensor can send its message directly to the sink. In this paper, we assume that a set of $K$ sinks are deployed within the area. In addition, we consider that the sensors are able to cooperate with each other in order to form a virtual antenna array to achieve a virtual MIMO communication system. This Virtual MIMO system is completely distributed since all the sensors are in different locations. Finally, we focus our study on a reporting-based application, where the sensors have to periodically report the same data related to their environment to the $\operatorname{sink}(\mathrm{s})$. This reporting period needs to be divided into two phases. The first phase is devoted to the channel learning/estimation in the TDD scenario. The channel estimation phase enables the sensors to optimize the distributed beamforming transmission. Basically, to estimate the channel, each receiver (sink) has to send a learning sequence. The second period is dedicated to the transmission phase. Within this period, the transmitters send the data to the $\operatorname{sink}(\mathrm{s})$, by adding, if necessary, a preprocessing of the information sent, such as a beamforming with the Hermitian transpose of the noisy estimate of the channel matrix acquired during the learning period.

It is clear that the efficiency of the system is related to the time spent in each period. To increase system performance, we need to increase the duration of the first period in order to have a good estimation of the channel matrix. Likewise, we also have to increase the duration of the second period in order to increase the global system bandwidth. We must obviously find a trade-off between the duration of each period. Another important issue is the number of sensors involved in the collaboration. It is clear that increasing the number of sensors will increase the bandwidth. However, this will also increase the amount of energy consumed by the network.

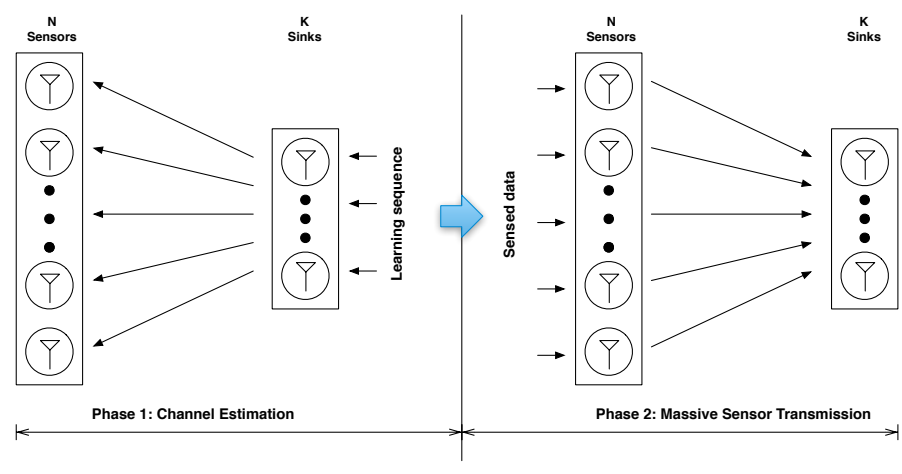

Fig. 1. Massive MIMO protocol.

\section{A. Training Period}

Let us consider a TDD system with a frame period denoted $T$. The sinks start by transmitting a training sequence of length $M$ data units. $M=\frac{\alpha T}{T_{s}}$, where $\alpha$ is the proportion of time devoted to the training phase and $T_{s}$ is the symbol of the transmission time $\left(T_{s} \sim \frac{1}{W}, W\right.$ is the bandwidth of the time symbol used). Then, the sensors start a transmission period equal to $(1-\alpha) T$.

During the training period the receivers have to transmit a training sequence. We assume that each sink $k$ transmits a training sequence noted as $\underline{\boldsymbol{c}}_{k}$ to the $N$ sensors. All the training sequences (i.e. $\underline{\boldsymbol{c}}_{1}, \underline{\boldsymbol{c}}_{2}, \cdots, \underline{\boldsymbol{c}}_{K}$ ) are orthogonal. The size of each training sequence is equal to $M$ symbols. The training sequence received by each transmitter from the $K$ sinks can be noted as $\boldsymbol{C}=\left[\begin{array}{llll}\underline{\boldsymbol{c}}_{1} & \underline{\boldsymbol{c}}_{2} & \cdots & \underline{\boldsymbol{c}}_{K}\end{array}\right]$, such that : $\boldsymbol{C}^{H} \boldsymbol{C}=M \cdot \mathbf{I d}$.

During the training period the signal received at the transmitters can be formulated as follows: 


\section{B. Transmission Period}

$\boldsymbol{Y}=\left[\begin{array}{ccc}y_{1}(1) & \cdots & y_{1}(M) \\ y_{2}(1) & \cdots & y_{2}(M) \\ \vdots & \ddots & \vdots \\ y_{N}(1) & \cdots & y_{1}(M)\end{array}\right]$

$\boldsymbol{Y}=\left[\begin{array}{ccc}h_{1,1} & \cdots & h_{1, K} \\ h_{2,1} & \cdots & h_{2, K} \\ \vdots & \ddots & \vdots \\ h_{N, 1} & \cdots & h_{N, K}\end{array}\right] \cdot\left[\begin{array}{c}\underline{\boldsymbol{c}}_{1}^{T} \\ \underline{\boldsymbol{c}}_{2}^{T} \\ \vdots \\ \underline{\boldsymbol{c}}_{K}^{T}\end{array}\right]+\left[\begin{array}{ccc}n_{1,1} & \cdots & n_{1, M} \\ n_{2,1} & \cdots & n_{2, M} \\ \vdots & \ddots & \vdots \\ n_{N, 1} & \cdots & h_{N, M}\end{array}\right]$

We note

$\boldsymbol{H}^{T}=\left[\begin{array}{ccc}h_{1,1} & \cdots & h_{1, K} \\ h_{2,1} & \cdots & h_{2, K} \\ \vdots & \ddots & \vdots \\ h_{N, 1} & \cdots & h_{N, K}\end{array}\right]$, and $\boldsymbol{N}=\left[\begin{array}{ccc}n_{1,1} & \cdots & n_{1, M} \\ n_{2,1} & \cdots & n_{2, M} \\ \vdots & \ddots & \vdots \\ n_{N, 1} & \cdots & h_{N, M}\end{array}\right]$

as the transfer matrix and the the noise matrix respectively.

For a given sensor (say sensor 1), we can write the received signal from $K$ sinks as follows

$$
\begin{array}{r}
{\left[y_{1}(1), \cdots, y_{1}(M)\right]=\left[h_{1,1}, \cdots, h_{1, K}\right] \cdot \boldsymbol{C}^{T}+} \\
{\left[n_{1,1}, \cdots, n_{1, M}\right]}
\end{array}
$$

Note that each sensor needs to know the whole code matrix $C$, since each receiver has to do the same processing. We assume that the matrix $C$ is a known matrix, thus there is no need for any cooperation between the sensors. The receivers can then estimate the channel as follows

$$
\begin{aligned}
{\left[\widehat{h}_{1,1}, \cdots, \widehat{h}_{1, K}\right]=} & \frac{1}{M} \cdot\left[y_{1}(1), \cdots, y_{1}(M)\right] \cdot \boldsymbol{C}^{*} \\
= & \frac{1}{M} \cdot\left[h_{1,1}, \cdots, h_{1, K}\right] \cdot \boldsymbol{C}^{T} \cdot \boldsymbol{C}^{*} \\
& +\frac{1}{M} \cdot\left[n_{1,1}, \cdots, n_{1, M}\right] \cdot \boldsymbol{C}^{*} \\
= & {\left[h_{1,1}, \cdots, h_{1, K}\right] } \\
& +\left[b_{1,1}, \cdots, b_{1, K}\right]
\end{aligned}
$$

where $\left[b_{1,1}, \cdots, b_{1, K}\right]=\underline{\boldsymbol{b}}$ is the error vector. It is a Gaussian vector of $K$ random variables of mean zero. We can compute the second moment of this error:

$$
\begin{aligned}
E\left[\underline{\boldsymbol{b}}^{H} \underline{\boldsymbol{b}}\right] & =\frac{1}{M^{2}} \cdot E\left[\boldsymbol{C}^{T} \underline{\boldsymbol{n}}^{H} \underline{\boldsymbol{n}} \boldsymbol{C}^{*}\right] \\
& =\frac{1}{M^{2}} \cdot \boldsymbol{C}^{T} \sigma^{2} \cdot \mathbf{I d} \cdot \boldsymbol{C}^{*} \\
& =\frac{\sigma^{2}}{M} \cdot \mathbf{I d}
\end{aligned}
$$
follows

Therefore, the estimated channel can be formulated as

$$
\forall i \in\{1, \cdots, N\} \forall j \in\{1, \cdots, K\} \quad \widehat{h}_{i, j}=h_{i, j}+b_{i, j},
$$

where $b_{i, j} \sim \mathcal{N}\left(0, \frac{\sigma^{2}}{M}\right)$. In the following we note $\widehat{\boldsymbol{H}}$ the $K$ lines and $N$ columns matrix and $\widehat{\boldsymbol{H}}=\left[\widehat{h}_{i, j}\right]$. we suppose that all the sensors measure the same data $s$. They also do not cooperate and each sensor knows only its channel to the $K$ sinks (i.e. not the channels of the other sensors to the $K$ sinks). The sensor will exploit the TDD protocol and channel reciprocity to beam-form the sensed data. The received signal at the sinks can be formulated as follows:

$$
\left[\begin{array}{c}
r_{1} \\
\vdots \\
r_{K}
\end{array}\right]=\left[\begin{array}{ccc}
h_{1,1} & \cdots & h_{N, 1} \\
\vdots & \ddots & \vdots \\
h_{1, K} & \cdots & h_{N, K}
\end{array}\right]\left[\begin{array}{c}
x_{1} \\
\vdots \\
x_{N}
\end{array}\right]+\left[\begin{array}{c}
z_{1} \\
\vdots \\
z_{K}
\end{array}\right]
$$

where $x_{i}$ is the data sent by sensor $i$. In our case, the transmitted data $x_{i}$ are computed as follows:

$$
\left[\begin{array}{c}
x_{1} \\
\vdots \\
x_{N}
\end{array}\right]=\frac{1}{\sqrt{K}} \widehat{\boldsymbol{H}}^{*}\left[\begin{array}{c}
s \\
\vdots \\
s
\end{array}\right]
$$

Thus we have for instance :

$$
x_{1}=\frac{1}{\sqrt{K}} \sum_{i=1}^{K} \widehat{h}_{1, i}^{*} s
$$

Therefore,

$$
\left[\begin{array}{c}
r_{1} \\
\vdots \\
r_{K}
\end{array}\right]=\frac{1}{\sqrt{K}} \boldsymbol{H}^{T} \widehat{\boldsymbol{H}}^{*}\left[\begin{array}{c}
s \\
\vdots \\
s
\end{array}\right]+\left[\begin{array}{c}
z_{1} \\
\vdots \\
z_{K}
\end{array}\right]
$$

Asymptotically, if $N \rightarrow \infty$ and $K$ is fixed and $h_{i, j} \sim \mathcal{N}(0,1)$.

$$
\frac{1}{N} \boldsymbol{H}^{T} \boldsymbol{H}^{*} \rightarrow \frac{1}{N}\left[\begin{array}{ccc}
N & \cdots & 0 \\
\vdots & \ddots & \vdots \\
0 & \cdots & N
\end{array}\right]=\mathbf{I d}
$$

and therefore in the asymptotic setting, we have for $j \in\{1, \cdots, K\}$ :

$$
r_{j}=\frac{N}{\sqrt{K}} s+z_{j}
$$

Thus, each sink is able to get the data without any interferences from the other receivers. This supposes of course that the channel is perfectly known but provides a guideline as to how the sensors should perform distributed beam-forming. Unfortunately, in practice, we have for $m \in\{1, \cdots, K\}$ : 


$$
\begin{aligned}
r_{m} & =\frac{1}{\sqrt{K}}\left(\left[h_{1, m}, \cdots, h_{N, m}\right]\right) \widehat{\boldsymbol{H}}^{*}\left[\begin{array}{c}
s \\
\vdots \\
s
\end{array}\right]+z_{m} \\
& =\frac{1}{\sqrt{K}}\left(\left[h_{1, m}, \cdots, h_{N, m}\right]\right) \widehat{\boldsymbol{H}}^{*}\left[\begin{array}{c}
\sum_{j=1}^{K} \widehat{h}_{1, j}^{*} \\
\vdots \\
\sum_{j=1}^{K} \widehat{h}_{N, j}^{*}
\end{array}\right]+z_{m}
\end{aligned}
$$

Therefore:

$$
\begin{aligned}
r_{m}= & \frac{1}{\sqrt{K}}\left[h_{1, m} \sum_{j=1}^{K} \widehat{h}_{1, j}^{*}+\cdots+\right. \\
& \left.h_{N, m} \sum_{j=1}^{K} \widehat{h}_{N, j}^{*}\right] \cdot s+z_{m}
\end{aligned}
$$

This results in:

$$
r_{m}=\frac{1}{\sqrt{K}}\left(\left|h_{1, m}\right|^{2}+\cdots+\left|h_{N, m}\right|^{2}\right) \cdot s+v_{m}+z_{m}
$$

where:

$$
v_{m}=\left[\sum_{i=1}^{N} h_{i, m} \sum_{j=1}^{K} b_{i, k}^{*}+\sum_{i=1}^{N} h_{i, m} \sum_{\substack{j=1 \\ j \neq m}}^{K} h_{i, j}^{*}\right] s
$$

Using Shannon's formula, the rate is then equal to:

$$
(1-\alpha) \log \left(1+\frac{\frac{P}{K}\left(\sum_{i=1}^{N}\left|h_{i, m}\right|^{2}\right)^{2}}{E\left[\left|v_{m}\right|^{2}\right]+\sigma^{2}}\right) .
$$

In the following, we assume that the fading is Rayleigh and thus $h_{i, k} \sim \mathcal{N}(0,1)$. We have:

$$
\begin{aligned}
E\left[\left|v_{m}\right|^{2}\right]=E[\mid & {\left.\left.\left[\sum_{i=1}^{N} h_{i, m} \sum_{j=1}^{K} b_{i, j}^{*}+\sum_{i=1}^{N} h_{i, m} \sum_{\substack{j=1 \\
j \neq m}}^{K} h_{i, j}^{*}\right) s\right|^{2}\right] } \\
E\left[\left|v_{m}\right|^{2}\right]= & E\left[|s|^{2}\right]\left[\left|\sum_{i=1}^{N} h_{i, m} \sum_{j=1}^{K} b_{i, j}^{*}\right|^{2}\right. \\
& +2 \Re\left(\left(\sum_{i=1}^{N} h_{i, m} \sum_{j=1}^{K} b_{i, j}^{*}\right)\left(\sum_{i=1}^{N} h_{i, m}^{*} \sum_{\substack{j=1 \\
j \neq m}}^{K} h_{i, j}\right)\right) \\
& \left.+\left|\sum_{i=1}^{N} h_{i, m} \sum_{\substack{j=1 \\
j \neq m}}^{K} h_{i, j}^{*}\right|^{2}\right] \\
E\left[\left|v_{m}\right|^{2}\right] & =P \frac{K \sigma^{2}}{M} \sum_{i=1}^{N}\left|h_{i, m}\right|^{2}+P\left|\sum_{i=1}^{N} h_{i, m} \sum_{\substack{j=1 \\
j \neq m}}^{K} h_{i, j}^{*}\right|^{2}
\end{aligned}
$$

Thus the total rate is equal to:

$$
\begin{aligned}
& R=(1-\alpha) \sum_{m=1}^{K} \log (1+ \\
& \left.\frac{\left.\left.\frac{P}{K}\left|\sum_{i=1}^{N}\right| h_{i, m}\right|^{2}\right|^{2}}{\frac{K \sigma^{2} T_{s}}{\alpha T} \sum_{i=1}^{N}\left|h_{i, m}\right|^{2}+\sigma^{2}+P\left|\sum_{i=1}^{N} h_{i, m} \sum_{\substack{j=1 \\
j \neq m}}^{K} h_{i, j}^{*}\right|^{2}}\right)
\end{aligned}
$$

In addition to the total rate, we compute the total energy consumed by the system which corresponds to the amount of energy consumed by the $K$ sinks during the training period (i.e. $\alpha T$ ) plus the amount of energy consumed by the sensors during the transmission period (i.e. $(1-\alpha) T)$ ). We can formulate the total energy consumed $E$ as follows:

$$
E=\alpha K P+(1-\alpha) P \sum_{i=1}^{N}\left(K \sigma^{2}+E\left(\left|\sum_{j=1}^{K} h_{i, j}\right|^{2}\right)\right.
$$

The problem we address is, given a maximum energy $E$ consumed, to find $\alpha, K$ and $N$ in order to maximize the total rate $R$.

\section{RESULTS}

We start our evaluation by analyzing the performances of the system with one sink (i.e. $K=1$ ). We fix the number of collaborating sensors, $N$, to 1000 and we study both the global rate and the energy consumption of the system. In Figure 2, we plot the global rate versus the period of time dedicated to the training period. As expected, the global rate is maximized for a given fraction of time dedicated to the training period $(8 \%$ in the figure). This is due to the fact that having $\alpha<0.08$ the sensors can not accurately estimate the channel, which results in a low throughput. On the other hand, increasing the training period $(\alpha>0.08)$, results in an accurate estimation of the channel, but also leads to a shorter transmission period, which also results in a low throughput. The optimal value is obtained for $\alpha^{*}=0.08$.

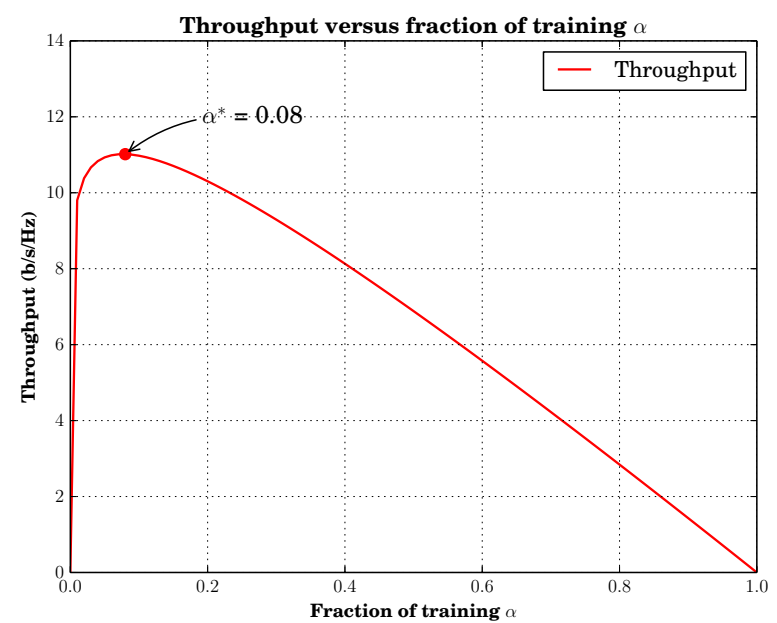

Fig. 2. Throughput versus fraction of training $\alpha$.

In Figure 3, we plot the maximal throughput that can be obtained when fixing the number of sinks to 1 and varying the number of sensors from 1 to 1000 . We can notice that the throughput increases as the number of sensors increases. However the more we increase $N$, the more marginal the potential gain becomes. However, increasing the number of collaborating sensors greatly increases the energy consumption. Indeed the energy consumption increases linearly with the number of sensors. 


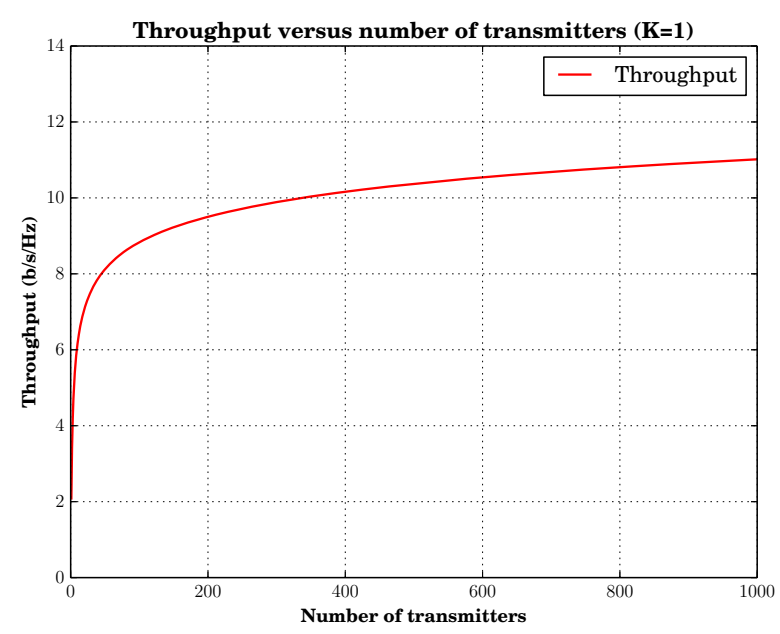

Fig. 3. Throughput versus Number of sensors.

In Figure 4, we plot the $K^{*}$ i.e. the number of sinks required to maximizes the total rate with a number of sensors equal to 200, 400, 600, 8001000 , respectively, and for a given maximum amount of energy available for each sensor. As we can observe, from a given number of sinks the global rate is maximized. It is not necessary to increase further the number of sinks.

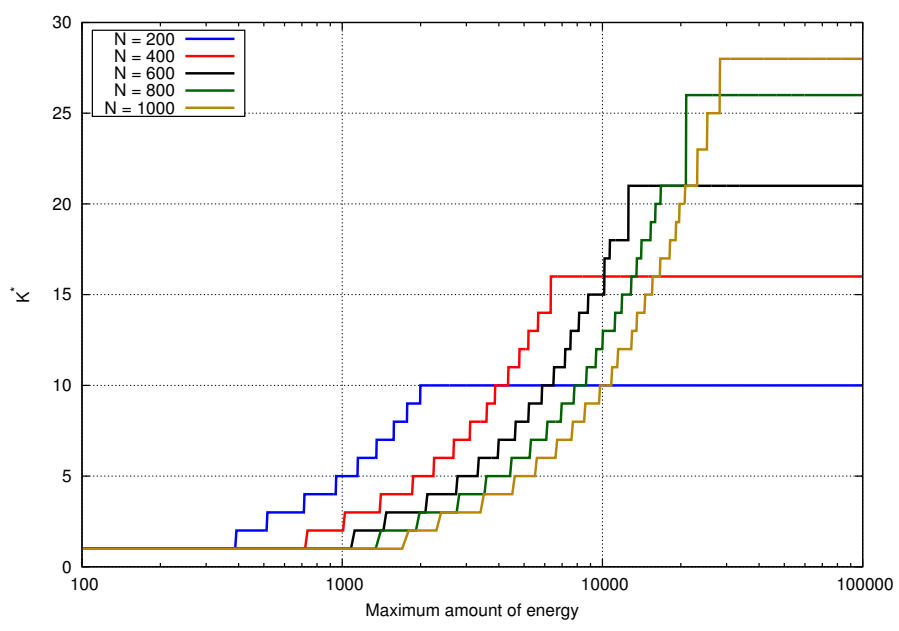

Fig. 4. Optimal number of sinks maximizing the total rate.

\section{CONCLUSION}

In this paper, we present a framework for massive MIMO cooperative communications for Wireless Sensor Networks. Our main objective is to analyze the performances of the deployment of a large number of sensors. This deployment should cope with a high demand for real time monitoring and should also take into account energy consumption. We have assumed a communications protocol with two phases: an initial training period followed by a second transmit period. The first period allows the sensors to estimate the channel state and the objective of the second period is to transmit the data sensed. In this paper, we analyzed the impact of the proportion of time devoted to each period. We study the throughput obtained with respect to the number of sensors when there is one sink. We also compute the optimal number of sinks with respect to the energy spent for different values of sensors. This work is a first step to establish a complete framework to study energy efficient Wireless Sensor Networks where the sensors collaborate to send information to a sink.

\section{REFERENCES}

[1] P. Vicaire, T. He, Q. Cao, T. Yan, G. Zhou, L. Gu, L. Luo, R. Stoleru, J. A. Stankovic, and T. F. Abdelzaher, "Achieving long-term surveillance in vigilnet," ACM Trans. Sen. Netw., vol. 5, no. 1, pp. 9:1-9:39, Feb. 2009. [Online]. Available: http://doi.acm.org/10.1145/1464420.1464429

[2] N. Xu, S. Rangwala, K. K. Chintalapudi, D. Ganesan, A. Broad, R. Govindan, and D. Estrin, "A wireless sensor network for structural monitoring," in Proceedings of the 2Nd International Conference on Embedded Networked Sensor Systems, ser. SenSys '04. New York, NY, USA: ACM, 2004, pp. 13-24. [Online]. Available: http://doi.acm.org/10.1145/1031495.1031498

[3] G. Werner-Allen, P. Swieskowski, and M. Welsh, "Motelab: a wireless sensor network testbed," in Information Processing in Sensor Networks, 2005. IPSN 2005. Fourth International Symposium on, April 2005, pp. 483-488.

[4] G. Barrenetxea, F. Ingelrest, G. Schaefer, M. Vetterli, O. Couach, and M. Parlange, "Sensorscope: Out-of-the-box environmental monitoring," in Information Processing in Sensor Networks, 2008. IPSN '08. International Conference on, April 2008, pp. 332-343.

[5] P. Dutta, J. Hui, J. Jeong, S. Kim, C. Sharp, J. Taneja, G. Tolle, K. Whitehouse, and D. Culler, "Trio: enabling sustainable and scalable outdoor wireless sensor network deployments," in Information Processing in Sensor Networks, 2006. IPSN 2006. The Fifth International Conference on, 2006, pp. 407-415.

[6] J. Chen, Z. Yin, D. Li, and T. Sun, "A distributed and effective cluster routing protocol of sensor networks," in Intelligent Networks and Intelligent Systems, 2008. ICINIS '08. First International Conference on, Nov 2008, pp. 271-275.

[7] V. T. Quang and T. Miyoshi, "Adaptive routing protocol with energy efficiency and event clustering for wireless sensor networks." IEICE Transactions, vol. 91-B, no. 9, pp. 2795-2805, 2008.

[8] L. Yuan, X. Wang, J. Gan, and Y. Zhao, "A data gathering algorithm based on mobile agent and emergent event-driven in cluster-based wsn." $J N W$, vol. 5, no. 10, pp. 1160-1168, 2010.

[9] W. Ge, J. Zhang, and G. Xue, "Joint clustering and optimal cooperative routing in wireless sensor networks," in Communications, 2008. ICC '08. IEEE International Conference on, May 2008, pp. 2216-2220.

[10] N. Dimokas, D. Katsaros, and Y. Manolopoulos, "Energyefficient distributed clustering in wireless sensor networks," Journal of Parallel and Distributed Computing, vol. 70, no. 4 , pp. $371-383,2010 . \quad$ [Online]. Available: http://www.sciencedirect.com/science/article/pii/S074373150900149X

[11] J. Hoydis, S. ten Brink, and M. Debbah, "Massive mimo in the $\mathrm{ul} / \mathrm{dl}$ of cellular networks: How many antennas do we need?" Selected Areas in Communications, IEEE Journal on, vol. 31, no. 2, pp. 160-171, February 2013.

[12] T. Marzetta, "Noncooperative cellular wireless with unlimited numbers of base station antennas," Wireless Communications, IEEE Transactions on, vol. 9, no. 11, pp. 3590-3600, November 2010.

[13] D. Gesbert, H. Bolcskei, D. Gore, and A. Paulraj, "Outdoor mimo wireless channels: models and performance prediction," Communications, IEEE Transactions on, vol. 50, no. 12, pp. 1926-1934, Dec 2002.

[14] I. E. Telatar, "Capacity of multi-antenna gaussian channels," European Transactions on Telecommunications, vol. 10, pp. 585-595, 1999. 